[23] R. Childs, J. Fortuna, J. Geneczko, and S. J. Fonash, in Proc. 12th IEEE Photo. Spec. Conf., p. 862, 1976.

[24] A. H. M. Kipperman, S. C. M. Backerra, H. J. Maaskamp, and R. J. C. van Zolingen, in Proc. 1st C.E.C. Photovoltaic Solar Energy Conf., p. 961, 1977.

[25] M. A. Green and C. Vithsupalert, to be published.

[26] M. A. Green and R. B. Godfrey, Appl. Phys. Lett., vol. 29, p. $610,1976$.

[27] P. Panayotatos,H. C. Card, and E. S. Yang, in Proc. 12th IEEE Photo. Spec. Conf., p. $920,1976$.

[28] S. Kar, D. Shankar, and S. P. Joshi, Extended Abstr. 1173, presented at the Int. Solar Energy Conf., International Solar Energy Soc., New Delhi, India, 1978.

[29] E. J. Charlson and J. C. Lien, J. Appl. Phys., vol. 46, p. 3982, 1975.

[30] R. J. Stirn and Y. C. M. Yeh, Appl. Phys. Lett., vol. 27, p. 95, 1975.

[31] J. P. Ponpon, R. Stuck, and P. Siffert, in Proc. 12th IEEE Photo. Spec. Conf., p. 900, 1976.

[32] A. H. M. Kipperman and M. H. Omar, Appl. Phys. Lett., vol. 28, p. 620,1976 .

[33] A. H. M. Kipperman, S. C. Backerra, and H. J. Maaskamp, in Proc. 1st C.E.C. Photovoltaic Solar Energy Conf., p. 956, 1977.

[34] D. R. Lillington and W. G. Townsend, Appl. Phys. Lett., vol. 28, p. 97,1976

[35] W. A. Anderson, S. M. Vernon, A. E. Delahoy, J. K. Kim, and P. Mathe, J. Vac. Sci. Tech., vol. 13,p. 1158, 1976.

[36] W. G. Townsend and D. R. Lillington, in Proc. 1st C.E.C. Photovoltaic Solar Energy Conf., p. 207, 1977.

[37] H. Matsumami, S. Matsumoto, and T. Tanako, in Proc. 12th IEEE Photo. Spec. Conf., p. 917, 1976.

[38] P. van Halen, R. E. Thomas, and R. van Overstraeten, in Proc. 12th IEEE Photo. Spec. Conf., p. 907, 1976.

[39] M. A. Green, R. B. Godfrey, and L. W. Davies, in Proc. 12th IEEE Photo. Spec. Conf., p. 896, 1976.

[40] E. J. Charlson, Final Rep., NSF Contract ENG 74-02918, Aug. 1976.

[41] R. J. Stirn, Y. C. M. Yeh, E. Y. Wang, F. P. Ernest, and C. J. Wu, in Tech. Dig. IEEE Int. Electron Devices Meet., p. 48, 1977.

[42] R. J. Stirn and Y. C. M. Yeh, in Proc. 12th IEEE Photo. Spec.
Conf., p. 883,1976 .

[43] J. P. Ponpon, J. J. Grob, A. Grob, R. Stuck, and P. Siffert, in Proc. 3rd Int. Conf. Ion Beam Analysis, 1977 (to be published).

[44] W. A. Anderson, Quarterly Rep., NSF Grant AER73-03197, May 1977.

[45] J. P. Ponpon and P. Siffert, J. Appl. Phys., vol. 47, p. 3248 , 1976.

[46] R. J. Stirn, presentation at ERDA Semiannu. Rev. Meet., Golden, CO, Oct. 1977 (unpublished).

[47] E. J. Charlson, private communication, Oct. 1977.

[48] S. Kar, in Proc. 12th IEEE Photo. Spec. Conf., p. 922, 1976.

[49] J. A. St. Pierre, R. Singh, J. Shewchun, and J. J. Loferski, in Proc. 12th IEEE Photo. Spec. Conf., p. 847, 1976.

[50] A. E. Delahoy, W. A. Anderson, and J. K. Kim, in Proc. 1st C.E.C. Photovoltaic Solar Energy Conf., p. 308, 1977.

[51] P. van Halen, R. Mertens, R. van Overstraeten, R. E. Thomas, and J, van Meerbergen, in Proc. 1st C.E.C. Photovoltaic Solar Energy Conf., p. 280, 1977.

[52] W. A. Anderson, J. K. Kim, and A. E. Delahoy, IEEE Trans. Electron Devices, ED.24, p. 453, 1977.

[53] W. A. Anderson and A. E. Delahoy, private communication, Nov. 1977.

[54] F. A. Lindholm, A. Neugroshel, C. T. Sah, M. P. Godlewski, and H. W. Brandhorst Jr., in Proc. 12th IEEE Photo. Spec. Conf., p. 1, 1976

[55] M. P. Godlewski, H. W. Brandhorst, Jr., and C. R. Baraona, in Proc. 11th IEEE Photo. Spec. Conf., p. 32, 1975.

[56] M. A. Green, private communication, Sept. 1977.

[57] R. J. Stirn, private communication, Aug. 1977.

[58] G. S. Kamath, J. Ewan, and R. C. Knechtli, in Proc. 12th IEEE Photo. Spec. Conf., p. 929, 1976.

[59] W. D. Johnston, J., and W. M. Callahan, in Proc. 12th IEEE Photo. Spec. Conf., p. 934, 1976.

[60] R. J. Stirn and Y. C. M. Yeh, in Proc. 11th IEEE Photo. Spec. Conf., p. 391, 1975

[61] S. Shevenock, S. J. Fonash, and J. Geneczko, in Tech. Dig. IEEE Int. Electron Devices Meet., p. 211, 1975.

[62] R. B. Godfrey and M. A. Green, Appl. Phys. Lett., vol. 31, p. $705,1977$.

[63] A. E. Delahoy, private communication, Sept. 1977.

\title{
$C-V$ Profiling of GaAs FET Films
}

\author{
JOHN D. WILEY
}

\begin{abstract}
The depletion layer capacitance of a Schottky barrier on a GaAs FET film can only be measured in series with the resistance of the undepleted portion of the film. This inherent series resistance may be significant at all values of bias and causes large errors in $C$ - $V$ profile determinations. By treating the depletion layer capacitance and the series resistance as a distributed $R C$ transmission line, it is possible to define an effective series resistance which can be related directly to the resistivity of the film. Using parameters typical of epitaxial films grown for GaAs FET applications, general criteria are developed for the profilability of these films. It is shown that, in general, films with small pinchoff voltages (i.e., films intended for low-noise FET applications)
\end{abstract}

Manuscript received April 3, 1978; revised May 2, 1978.

The author is with the Department of Electrical and Computer Engineering, University of Wisconsin, Madison, WI 53706. are much more difficult to profile accurately than films with large pinchoff voltages.

\section{INTRODUCTION}

C APACITANCE-VOLTAGE $(C-V)$ profiling [1]-[4] is probably the single most important method used to characterize GaAs films grown on semi-insulating substrates for MESFET [5] applications. The reasons for the wide acceptance of this measurement technique are clear: 1) many common metals form excellent Schottky barriers on GaAs without elaborate surface preparation, passivation, or guarding; 2) one relatively simple measurement provides the material parameters of greatest interest to the crystal grower and de- 
vice designer (film thickness, doping level, uniformity, and pinchoff voltage); and 3) commercial profiling instruments aris available which, under favorable circumstances, eliminate the need for any calculations, corrections, or data reduction.

Unfortunately, the validity of the $C-V$ profiling method does not rest on any rigorous analysis but, rather, on a number of simpliflying assumptions which lead to the equations

$$
N(d)=-\frac{C^{3}}{q \epsilon A^{2}}\left(\frac{d C}{d V}\right)^{-1}
$$

and

$$
d=\frac{\epsilon A}{C}
$$

where $C$ is the depletion-layer capacitance at reverse bias $V$, $q$ is the electronic charge, $\epsilon$ is the semiconductor dielectri: constant, $A$ is the diode area, $d$ is the depletion depth, and $N(d)$ is the doping level at the depth $d$. A number of authors [6]-[13] have discussed the model assumptions which under. lie (1) and (2), and the conditions under which real diodes will violate these assumptions. From a practical point of view, the central assumption of $C-V$ profiling is clearly that (1) and (2) can be used in the following simple way: one measures $C(V)$, assumes that the measured $C$ is indeed the depletion-layer capacitance, and then calculates $N(d)$. In an earlier pape: [11] Wiley and Miller emphasized that when profiling $\mathrm{GaA}$; FET films one must not automatically assume that the mea. sured capacitance is simply the depletion-layer capacitance. As can be seen from Fig. 1, the depletion-layer capacitance is not directly accessible, and can be measured only in series with the lateral resistance of the undepleted portion of the epitaxial film. Under these circumstances, the measured capacitanct $C^{\prime}$ is given approximately by

$$
C^{\prime} \approx \frac{C}{1+\omega^{2} R^{2} C^{2}}
$$

where $C$ is the true depletion-layer capacitance, $R$ is the serie; resistance, and $\omega$ is the measurement frequency. As pinchof: is approached, $R$ becomes very large, and the direct use o: $C^{\prime}$ in (1) and (2) will obviously result in profiling errors. Several examples of profiles distorted by series-resistance effects were given in [11]. As discussed in [11], (3) is basec on a simple lumped-element equivalent circuit in which the entire depletion-layer capacitance is placed in series with : single equivalent resistance $R$. For typical film thicknes: $(<1 \mu \mathrm{m})$ and diode diameters $(>100 \mu \mathrm{m})$ this is clearly : crude approximation, and the diode should be modeled by : distributed $R C$ network. It is the purpose of this paper to report some of the consequences of the distributed network analysis as they relate specifically to the $C-V$ profiling $o$ : GaAs FET films. It will be shown that the distributed analysi: yields a dimensionless parameter $\omega R_{e} C$, where $R_{e}$ is an effec. tive series resistance which can be related to the resistivity o: the film. The magnitude of $\omega R_{e} C$ varies with dc bias, diverg ing as pinchoff is approached. As long as $\omega R_{e} C$ remains smal over a significant range of $\mathrm{dc}$ bias values, however, accuratt profiling is possible. This condition is shown to be most easily achieved for films with large pinchoff voltages. In the low.

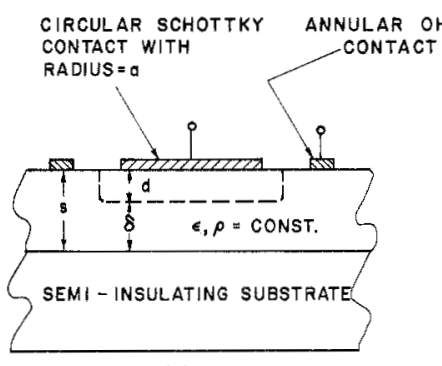

(a)

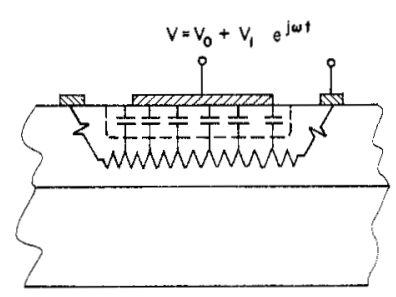

(b)
Fig. 1. (a) Cross-sectional view of the diode structure analyzed in this paper, showing a uniformly doped epitaxial GaAs film of thickness $s$ on a nonconducting substrate; a circular Schottky contact of radius $a$; a depletion layer of thickness $d$; and a conducting channel of undepleted epitaxial material of thickness $\delta$. The ohmic contact is an annular ring. (b) A distributed $R C$ equivalent circuit of the structure shown in (a). The small resistance between the ohmic contact and the channel beneath the depletion layer is assumed to be negligible.

frequency or low $R_{e}$ limits, (3) is recovered from the distributed analysis, validating the conclusions reached in [11].

\section{ANALYSIS}

Consider a circular Schottky-barrier contact of radius " $a$ " on a GaAs FET film (uniform doping level $N_{d}$; film thickness $s$; semi-insulating substrate) as shown in Fig. 1(a). In order to preserve the symmetry of the circular contact and minimize series resistance to the greatest extent possible, it will be assumed that ohmic contact to the epitaxial film is made via a closely spaced annular contact of low contact resistance. In the depletion approximation, the total depletion-layer capacitance is then given by

$$
C=\frac{\epsilon A}{d} \text {. }
$$

Although edge effects [10] are indicated schematically in Fig. 1 by extending the depletion layer beyond the edge of the Schottky contact, it must be remembered that Fig. 1 is drawn grossly out-of-scale, with the film thickness exaggerated by at least two orders of magnitude. Assuming, for now, that edge effects are negligible, we take $A=\pi a^{2}$. The thin layer of conductive film sandwiched between the depletion layer and the substrate is of thickness $\delta$ and resistivity $\rho$. It, therefore, has a uniform sheet resistance

$$
R_{s}=\frac{\rho}{\delta} \text {. }
$$

Any given value of $\mathrm{dc}$ bias between the Schottky and ohmic contacts will specify unique values for $C$ and $R_{s}$. Clearly, as the reverse bias is increased in magnitude, $C$ will decrease and $R_{s}$ will increase. Because of the distributed nature of the structure, however, neither $C$ nor $R_{s}$ is independently measureable, and we must determine the terminal impedance of the equivalent circuit shown in Fig. 1(b).

Problems which are mathematically similar [14] or identical [15] - [17] to the present one are well known, so details of the solution will be omitted. By considering differential segments of the distributed network of Fig. 1(b), it is straightforward to show that the ac current and voltage must satisfy the equations 


$$
\frac{d I(r)}{d r}=j \frac{2 \pi \epsilon \omega}{d} r V(r)
$$

and

$$
I(r)=\frac{2 \pi \delta}{\rho} r \frac{d V(r)}{d r}
$$

where $r$ is the radial coordinate measured from the center of the Schottky contact. Differentiating (7) and equating the expressions for $d I(r) / d r$ gives

$$
r \frac{d^{2} V(r)}{d r^{2}}+\frac{d V(r)}{d r}-j \frac{\epsilon \omega \rho}{\delta d} r V(r)=0 .
$$

Defining the dimensionless coordinate

$$
x=\sqrt{\frac{\omega \epsilon \rho}{\delta d}} r
$$

(8) becomes

$$
\frac{d^{2} V(x)}{d x^{2}}+\frac{1}{x} \frac{d V(x)}{d x}-j V(x)=0
$$

which is the equation that must be solved for $V(x)$. Once $V(x)$ is known, $I(x)$ may be calculated using (7), and the complex impedance of the network obtained. Equation (10) is one form of Bessel's equation, this particular form having solutions which are known as Kelvin functions [18]. Taking the voltage to be finite for all $r \leqslant a$, and equal to the applied voltage at the terminals, one obtains

$$
V(x)=V_{1} \frac{\operatorname{ber}(x)+j \text { bei }(x)}{\text { ber }\left(x_{a}\right)+j \text { bei }\left(x_{a}\right)}
$$

where $x_{a}$ is the value of $x$ at $r=a$

$$
x_{a}=\sqrt{\frac{\omega \epsilon \rho}{\delta d}} a
$$

and the functions ber $(x)$ and bei $(x)$ are tabulated Kelvin functions [18]. Substituting (11) into (7) gives

$$
I(x)=\frac{2 \pi \delta V_{1} x}{\rho} \frac{\text { ber }^{\prime}(x)+j \text { bei }^{\prime}(x)}{\operatorname{ber}\left(x_{a}\right)+j \text { bei }\left(x_{a}\right)}
$$

where primes indicate differentiation with respect to $x$. The complex admittance at the terminals is then given by

$$
Y=\frac{I_{\text {total }}}{V_{\text {applied }}}=\frac{I\left(x_{a}\right)}{V_{1}}
$$

or

$$
Y=\frac{2 \pi \delta x_{a}}{\rho}\left[A_{1}+j A_{2}\right]=\frac{x_{a}}{4 R_{e}}\left[A_{1}+j A_{2}\right]
$$

where

$$
\begin{aligned}
& A_{1}=\frac{\operatorname{ber}\left(x_{a}\right) \text { ber' }^{\prime}\left(x_{a}\right)+\operatorname{bei}\left(x_{a}\right) \text { bei' }\left(x_{a}\right)}{\operatorname{ber}^{2}\left(x_{a}\right)+\operatorname{bei}^{2}\left(x_{a}\right)} \\
& A_{2}=\frac{\operatorname{ber}\left(x_{a}\right) \operatorname{bei}^{\prime}\left(x_{a}\right)-\operatorname{bei}\left(x_{a}\right) \text { ber' }^{\prime}\left(x_{a}\right)}{\operatorname{ber}^{2}\left(x_{a}\right)+\operatorname{bei}^{2}\left(x_{a}\right)}
\end{aligned}
$$

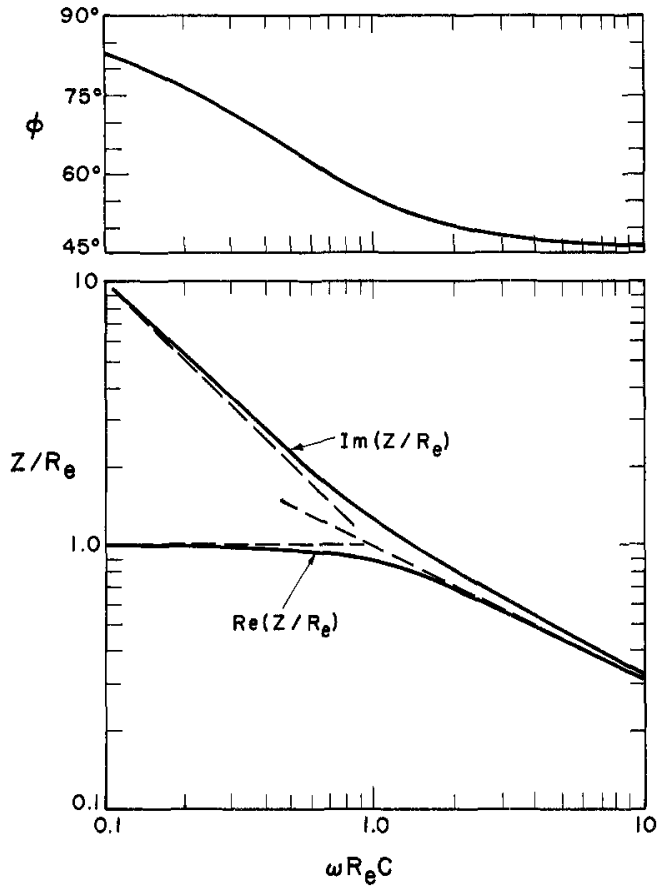

Fig. 2. Plots of the real and imaginary parts of the complex impedance of the distributed $R C$ network (normalized by $R_{e}$ ) and the phase angle as functions of $\omega R_{e} C$. The asy mptotic behavior of $Z$, shown by dashed lines, is discussed in the text.

and

$$
R_{e}=\frac{\rho}{8 \pi \delta} .
$$

The parameter $R_{e}$ is an effective sheet resistance the significance of which will be discussed below. In terms of these same parameters, the complex impedance and phase angle are given by

$$
\frac{Z}{R_{e}}=\frac{4 A_{1}}{x_{a}\left(A_{1}^{2}+A_{2}^{2}\right)}-j \frac{4 A_{2}}{x_{a}\left(A_{1}^{2}+A_{2}^{2}\right)}
$$

and

$$
\phi=\tan ^{-1}\left(A_{2} / A_{1}\right) .
$$

Equations (19) and (20) are shown in Fig. 2 as functions of $\omega R_{e} C$. The distributed $R C$ network is seen to behave exactly as expected: For $\omega R_{e} C \ll 1$, the capacitance is dominant so that $\operatorname{Im}(Z) \gg \operatorname{Re}(Z)$, and $\phi \rightarrow 90^{\circ}$. For $0.3 \leqslant \omega R_{e} C \leqslant 3$ distributed effects are dominant and the behavior of $Z$ and $\phi$ is complicated. For $\omega R_{e} C \geqslant 3$ the network approaches the perimeter-dominated regime which has been treated by Lehovec [17] for arbitrary geometries. In this regime the real and imaginary parts of the impedance make equal contributions and $\phi \rightarrow 45^{\circ}$.

Using series approximations given in [18], the following limits can be obtained:

For $\omega R_{e} C \ll 1$

$$
Z \longrightarrow R_{e}+\frac{1}{j \omega C}
$$




$$
\begin{aligned}
& \text { For } \omega R_{e} C \gg 1 \\
& Z \longrightarrow \sqrt{\frac{R_{e}}{\omega C}}(1-j) .
\end{aligned}
$$

Equation (21) is, of course, precisely the result that would be obtained for a lumped equivalent circuit with resistance $R=R_{e}$. Thus $R_{e}$ is the appropriate intrinsic resistance to use in the analysis given in [11]. (Here, the word "intrinsic" is used to denote the distributed resistance beneath the depletion layer, as distinct from any "extrinsic" series resistance such as the contact and spreading resistances of the ohmic contact. Such extrinsic resistance can be made negligibly small, whereals the intrinsic resistance is a significant and unavoidable con1. sequence of the structure.) The real and imaginary parts of (21) and (22) are shown in Fig. 2 as dashed lines.

In the intermediate $\left(\omega R_{e} C \approx 1\right)$ region where distributed effects are dominant, no simple approximations to $Z$ have been found. It is possible to use the series expansions given in [18] to cast $Z$ into a form which is interpretable as a succeision of lumped-element approximations containing $n$ lumped resistors and capacitors of suitably chosen values. In the lim.t $n \rightarrow \infty$, the distributed model is recovered. This approach. quickly leads (for any $n>2$ ) to lumped-element approximations which are more cumbersome than the distributed solution. At the present time it does not appear that any exact (or even approximate) inverse transform will be found whicn could allow one to generalize the simple $C-V$ profiling equitions and deduce $N_{d}(d)$ from $Z\left(V_{0}\right)$ to any greater accuracy than that discussed [11]. This problem is, however, still under investigation.

\section{Application to GaAs FET Films}

For purposes of this section, the ideal FET film will be take.1 to be one with uniform doping from the surface to the sut strate. ${ }^{1}$ The doping levels of interest are well within the range $5 \times 10^{15} \leqslant N_{d} \leqslant 5 \times 10^{17} \mathrm{~cm}^{-3}$, and film thicknesses are typically $<1 \mu \mathrm{m}$. A further restriction is provided by the fact that, for practical applications, pinchoff voltages should be in the range $2.8 \lesssim V_{p} \leqslant 10.8 \mathrm{~V}$ (obtained by adding a built-iin voltage of $0.8 \mathrm{~V}$ to the desired range of externally applied voltages at pinchoff: $2 \lesssim V_{p}^{e} \lesssim 10 \mathrm{~V}$ [5]). These parameters together with the results of Section II, allow the calculation of the important quantity $\omega R_{e} C$ which determines the feas:bility of conventional $C-V$ profiling.

For a uniformly doped FET film, the pinchoff voltage is given by

$$
V_{p}=\frac{q N_{d} s^{2}}{2 \epsilon}
$$

where $N_{d}$ is the doping level, $s$ is the film thickness, and $V_{p}$ is

\footnotetext{
${ }^{1}$ Actual FET films are usually more complicated than this, but should show nearly identical behavior in all characteristics that are of interest in this paper. Thus for example, any undoped or $\mathrm{Cr}$-doped "buffer layers" will be considered to be semi-insulating extensions of the sukstrate. Any thin-surface layers grown for contact improvement or breakdown control are assumed to be fully depleted at zero bias.
}

understood to include a built-in voltage of ${ }^{2} V_{b i} \approx 0.75 \pm$ $0.06 \mathrm{~V}$. The depletion-layer capacitance can be written in terms of $V_{p}$ as

$$
C=C_{m} \sqrt{\frac{V_{p}}{V}}
$$

where $C_{m}$ is the minimum possible value of $C$ (i.e., the value of $C$ at pinchoff)

$$
C_{m}=\frac{\epsilon A}{s}
$$

and $V$ is the dc reverse bias. Similarly, the effective sheet resistance $R_{e}$ can be written in terms of $V_{p}$

$$
R_{e}=\frac{\rho}{8 \pi \delta}=\frac{\rho}{8 \pi(s-d)}
$$

or

$$
R_{e}=\frac{R_{m}}{\left(1-\sqrt{V / V_{p}}\right)}
$$

where

$$
R_{m}=\frac{\rho}{8 \pi s} .
$$

Combining (24) and (27) and multiplying by $\omega$

$$
\omega R_{e} C=\frac{\omega R_{m} C_{m}}{\sqrt{\frac{V}{V_{p}}}\left(1-\sqrt{\frac{V}{V_{p}}}\right)} .
$$

The bias-dependent part of (29) is

$$
f(V)=\frac{1}{\sqrt{\frac{V}{V_{p}}}\left(1-\sqrt{\frac{V}{V_{p}}}\right)}
$$

which diverges as $V \rightarrow 0$ and as $V \rightarrow V_{p}$, and has a minimum value of $f(V)=4$ at $V=V_{p} / 4$. Since the depletion depth is proportional to $\sqrt{V}$, this implies that $\omega R_{e} C$ will assume its minimum value when the film is half depleted. The apparent divergence of $f(v)$ as $V \rightarrow 0$ is of no practical concern since $V=V_{b i}$ is the minimum useful value of $V$ in $C-V$ profiling ( $V=V_{b i}$ when the externally applied bias is 0 ). The divergence for $V \rightarrow V_{p}$, on the other hand, means that $\omega R_{e} C$ will always become large as pinchoff is approached. This, in turn, implies that FET films can never be profiled to full pinchoff. In practice, the conventional profiling equations become unacceptably inaccurate [11] when $\omega R_{e} C \gtrsim 0.2-0.3$. Thus it is of interest to determine the conditions under which $\omega R_{e} C$ can be held below this critical range.

$$
\begin{aligned}
& { }^{2} \text { This value of } V_{b i} \text { is based on the equation } \\
& V_{b i}=0.026\left[28.934+\ln \left(\frac{N_{d}}{5.1}\right)\right]
\end{aligned}
$$

( $N_{d}$ in units of $10^{16} \mathrm{~cm}^{-3} ; V_{b i}$ in volts) which, in turn, is an empirical fit to data given in [5]. This equation was incorrectly printed with a minus sign in [11]. 
At any given temperature, the parameters $R_{m}$ and $C_{m}$ depend only on the epitaxial film thickness, doping level, and dielectric constant. Combining (25) and (28)

$$
\omega R_{m} C_{m}=\frac{\omega \epsilon A \rho}{8 \pi s^{2}}
$$

From this equation it is seen that the doping level is important only to the extent that it affects $\rho$. In the range $5 \times 10^{15} \leqslant$ $N_{d} \leqslant 5 \times 10^{17} \mathrm{~cm}^{-3}$, $\rho$ for $\mathrm{n}-\mathrm{GaAs}$ at $300 \mathrm{~K}$ may be represented by the simple power-law expression ${ }^{3}$

$$
\rho(\Omega \cdot \mathrm{cm}) \approx \frac{1.5 \times 10^{15}}{N_{d}\left(\mathrm{~cm}^{-3}\right)} .
$$

When (32) is inserted into (31), the resulting $N_{d} s^{2}$ product in the denominator may be re-expressed in terms of $V_{p}$ using (23). Using $\epsilon=12.8 \epsilon_{0}$ for $\mathrm{GaAs}$, and converting to consistent MKS units for all quantities then gives

$$
\omega R_{m} C_{m}=0.478 \frac{\omega\left(\mathrm{s}^{-1}\right) A\left(\mathrm{~m}^{2}\right)}{V_{p}(\mathrm{~V})}
$$

It is important to note that, because of (32), $N_{d}$ and $s$ have entered only in the form $N_{d} s^{2}$ (i.e., in a form $\propto V_{p}$ ). The practical consequence of this is that low $-V_{p}$ films will always be more difficult to profile accurately than high $V_{p}$ films. This is true, regardless of how the specific value of $V_{p}$ was obtained (small $N_{d}$ and large $s$ or large $N_{d}$ and small s).

In order to illustrate practical values of $\omega R_{e} C$, Fig. 3 has been prepared using (29) and (33) for a measurement frequency of $1 \mathrm{MHz}$ and a diode area of $2.027 \times 10^{-7} \mathrm{~m}^{2}$ (corresponding to a circular diode of 20 -mil diameter). Using the criterion that $\omega R_{e} C$ must be $\lesssim 0.2$ for accurate profiling, it is seen that a film with $V_{p}=10 \mathrm{~V}$ could be profiled from zero bias to about 50-60 percent of pinchoff before seriesresistance errors become unacceptable. Films with smaller $V_{p}$ are totally unprofilable unless the measurement frequency and/or diode area are reduced.

The curves shown in Fig. 3 can be scaled to apply to other diode areas or measurement frequencies by shifting them rigidly upward or downward in proportion to $\omega A$. Thus for a 10-mil diameter diode, the curves are shifted downward by a factor of 4 . This results in $\omega R_{e} C<0.2$ for all $V<0.85 V_{p}$ for the $V_{p}=10 \mathrm{~V}$ film; and for all $V<0.65 V_{p}$ for the $V_{p}=5 \mathrm{~V}$ film. The $V_{p}=2 \mathrm{~V}$ film remains unprofilable, with $\omega R_{e} C \geqslant 0.3$ at all values of bias.

\section{Discussion}

Commercial capacitance meters and profiling instruments ${ }^{4}$ measure only the imaginary component of the RF current and assume it to be proportional to $j \omega C$.

\footnotetext{
${ }^{3}$ J. D. Wiley (unpublished compilation). Equation (31) takes into account the fact that lightly doped films tend to be more strongly compensated than heavily doped films.

${ }^{4}$ Manually balanced impedance bridges are excluded from consideration since they are too slow and cumbersome to use in routine $\mathrm{C}-\mathrm{V}$ profiling.
}

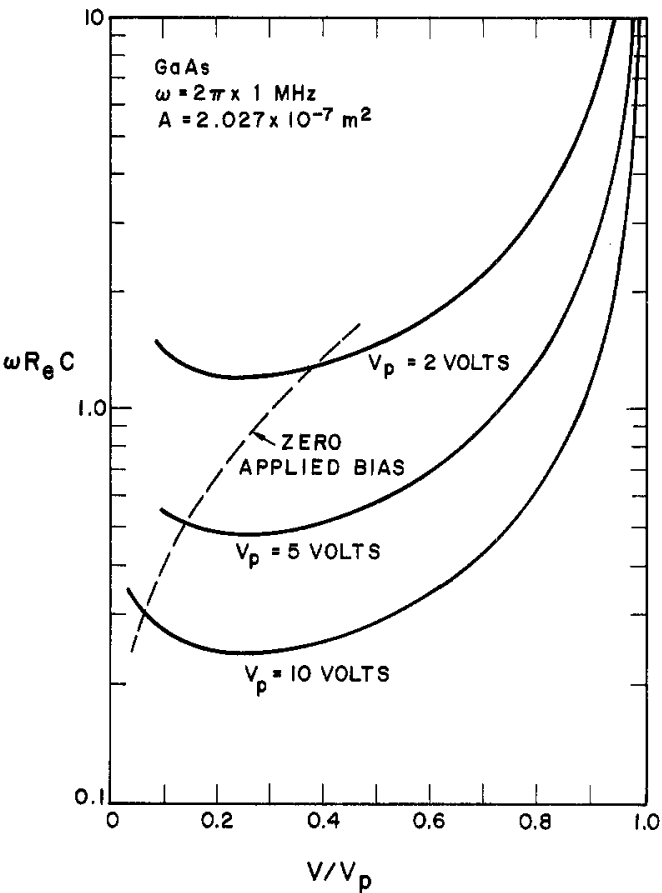

Fig. 3. Values of $\omega R_{e} C$ versus dc bias (normalized by the pinchoff voltage) for various values of pinchoff voltage. Points to the left of the dashed line correspond to forward bias and are irrelevant for profiling purposes. The chosen area corresponds to a circular diode 20 mils in diameter, and the measurement frequency is taken to be $1 \mathrm{MHz}$. The curves can be scaled for other values as discussed in the text.

In Fig. 2, the reactance of the true depletion-layer capacitance is shown by the upper-left dashed line (slope =-1). From this, it is seen that series-resistance effects will cause one to underestimate the magnitude of the depletion-layer capacitance and, hence, overestimate the depletion depth. Profile distortions will thus always include a shift toward larger depletion depths at any given value of dc bias. Further distortions will be caused by erroneous $N_{d}$ calculations and, as discussed in [11], these distortions result in complex profile shapes.

Fig. 4 shows a typical distorted profile which shall be taken as the prototype for mildly distorted profiles. A profile such as this would be observed in cases where $\omega R_{e} C \gtrsim 0.3$ near zero bias. As the reverse bias is increased, $C$ decreases more rapidly than $R_{e}$ increases, and $\omega R_{e} C$ drops below 0.2 over some range of bias values. In this region, reasonably accurate results are obtained. As pinchoff is approached, $R_{e}$ increases rapidly and $\omega R_{e} C$ again exceeds 0.3 , causing serious distortions. Four important features of the profile in Fig. 4 have been labeled A-D and will now be discussed in more detail.

A) The abrupt upturn in the profile near zero bias (which must not be confused with a similar artifact observed for all profiles in forward bias) is a characteristic feature which may be taken as conclusive evidence that series-resistance distortions are present, and that the entire profile is suspect. The absence of this feature, usually indicates that the early (lowbias) part of the profile is undistorted by series resistance.

B) Depending on the actual range of $\omega R_{e} C$ values for a given diode, the central portion of the profile may be reason- 


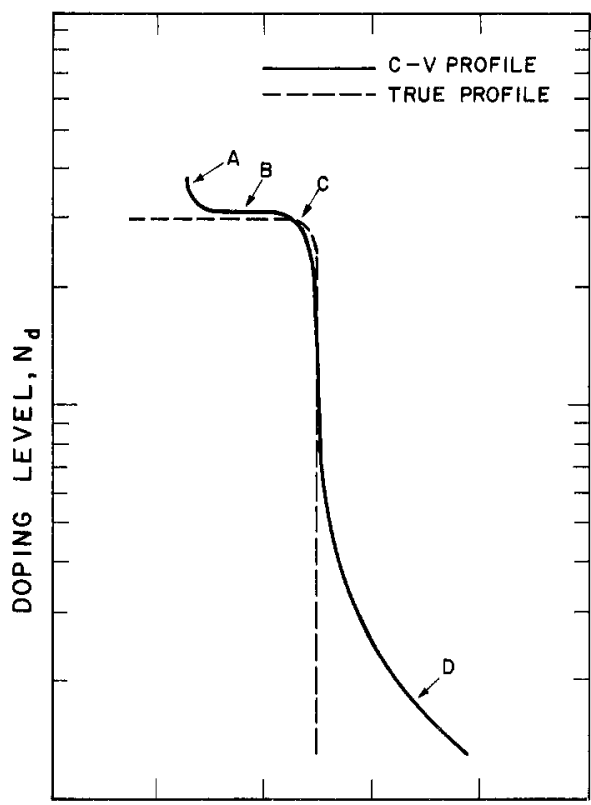

DEPLETION DEPTH, d

Fig. 4. Schematic illustration of typical distortions which are observed in $C-V$ profiles of GaAs FET films and are attributable to series resistance. Examples of more severe distortions can be found in [11]. Individual features A-D are discussed in the text.

ably accurate. Fig. 4 shows a small positive error in $N_{c l}$. Although depletion-depth errors are also present, they are not apparent when the profile is flat (uniform).

C) Under the conditions of mild distortion assumed hers, the "knee" region of the profile appears at a slightly smaller depletion depth and is more "rounded" than the true profils. Furthermore, the knee of the distorted profile occurs at a value of $d c$ bias which is significantly lower than the true pinchoff voltage of the film. This point is emphasized because many workers use the value of bias at the knee as the pincholf voltage. When transistors are fabricated on films which have been characterized in this manner, the transistors (having very narrow gates and, therefore, no series resistance artifacts) will appear to have anomalously large pinchoff voltages.

D) As pinchoff is approached, the errors in both $N_{d}$ and $d$ become extremely large, leading to a tail that appears to extend well into the substrate. This tail was discussed more fully in [11]. Lehovec [12] and Lehovec and Zuleeg [19] have given extremely interesting discussions of the constquences of a film-substrate depletion layer for $C-V$ profile results. They show that when the two depletion layers begin to merge, a tail similar to that shown in Fig. 4 results and tha :, in fact, the tail may even turn upward for larger values of reverse bias. If, as a first approximation, one assumes that any depletion layer at the substrate interface can be accounted for by simply reducing the effective thickness of the epitaxial film [5], then it is clear that series-resistance effects will set in before the depletion layers begin to merge, and the effects described by Lehovec and Zuleeg will be masked. This cortclusion will not hold, however, for the gate capacitance of an FET, for which series-resistance effects should be small.

It is important to note that the profile shown in Fig. 4 :s only mildly distorted in that it bears at least some resemblance to the true profile, and allows one to deduce approximately correct values for $N_{d}$ and $s$ without elaborate calculations or corrections. As shown in [11], series resistance effects can (and often do) lead to much worse distortions, resulting in profiles which bear no resemblance at all to the correct profile and which are, therefore, useless for quantitative purposes.

In [11], it was suggested that the most practical way to guard against profile distortions is to monitor the phase angle $\phi$ between the RF current and voltage during profiling. The distributed network analysis confirms this suggestion. From Fig. 4, it is seen that $\phi$ is extremely sensitive to small components of series resistance, deviating substantially from $90^{\circ}$ even for $\omega R_{e} C \leqslant 0.1$. Experimental measurements and computer simulations [11], [20] have shown that $C-V$ profiles of FET films are accurate to within 1 percent for $\phi>80^{\circ}$, but that the accuracy deteriorates very rapidly for $\phi<75^{\circ}$. For $\phi \lesssim 70^{\circ}$, the distributed nature of the $R C$ network becomes important, and the lumped analysis and correction factors given in [11] become unuseable.

An interesting feature of the distributed analysis is the prediction that for large values of $\omega R_{e} C$ the real and imaginary parts of the impedance become equal and the phase angle approaches $45^{\circ}$. This fact is of little practical consequence, however. It must be remembered that the analysis has included only the inherent or distributed component of series resistance. The presence of any additional parasitic resistance will shift the curve of $\operatorname{Re}\left(Z / R_{e}\right)$ rigidly upward in direct proportion to the magnitude of this excess resistance. This will cause $\operatorname{Re}(Z)$ to exceed $\operatorname{Im}(Z)$ at sufficiently high $\omega R_{e} C$, reduce $\phi$ at all values of $\omega R_{e} C$, and cause $\phi \rightarrow 0$ as $\omega R_{e} C \rightarrow \infty$. Since some amount of extrinsic resistance must always be present (ohmic contact resistance, for example), it is clear that, in practice, $\phi$ may assume any value between $0^{\circ}$ and $90^{\circ}$.

By monitoring $\phi$ during profile measurements, one can detect the presence of series resistance effects, but the problem of obtaining corrected profiles remains. Several possible remedies were discussed in [11], and that discussion will not be repeated here except to note that each measure taken to reduce the series-resistance distortions carries with it a new set of problems, uncertainties, and possible distortions. The simplest, and probably the safest, experimental remedy is to use diodes of the smallest area possible. Lower limits to the diode area will be set by the sensitivity of the measurement system, and the confidence with which one can make stray $C$ and edge-effect corrections. Using the results of Wasserstrom and McKenna [21], the edge-effect correction for the capacitance of a circular diode on $\mathrm{n}-\mathrm{GaAs}$ is

$$
C=\frac{\epsilon A}{d}\left(1+1.55 \frac{d}{a}\right)
$$

where $\epsilon A / d$ is the uncorrected capacitance, and $a$ is the diode radius. For a diode of 5 -mil diameter, the correction amounts to about 2.5 percent at $d=1 \mu \mathrm{m}$. If uncorrected, this would cause a (roughly) 8-percent error in the $N_{d}$ determination. Smaller diodes are, of course, correspondingly worse because of their smaller surface area-to-perimeter ratios. In some cases, it may be desirable to alter the geometry of the Schottky contact while keeping the area at some reasonably small fixed 
value. If, for example, series resistance effects are severe for a 10-mil diameter diode, one could use an elongated diode with 5-mil diameter semicircular ends and a 12-mil-long joining segment. This diode would have the same area as the 10-mil circular diode, a 75-percent larger perimeter, and would show a four-fold reduction in series-resistance effects. The small increase in edge-effect errors is more than offset by reduced series resistance. Similar results could be achieved by bonding together several small circular diodes and measuring them in parallel.

\section{CONCLUSIONS}

Of the many artifacts which can affect the accuracy of $C-V$ profiles, series resistance effects are, for FET films, the most common and the most severe. A distributed series resistance is an inherent feature of these films, and its effects must always restrict the range of accurate profiling to something less than the full film thickness. As was shown in Section III, intrinsic series-resistance effects become more severe, the lower the pinchoff voltage of the film. This fact should be of particular interest to those engaged in the fabrication of lownoise GaAs FET's for microwave preamplifier applications, since such devices are usually designed for low $(<4 . \mathrm{V})$ pinchoff. Even when distortions in the shape of the profile are relatively minor, series resistance can cause large errors in the apparent pinchoff voltage. Again, the errors are doubly serious for low $V_{p}$ material, being both more likely to occur and larger as a fractional error.

Some of the experimental precautions which should be taken when profiling GaAs FET films were discussed in Section III. In addition to taking these precautions, it is prudent (for low $-V_{p}$ material, vital) that the $C-V$ measurements be supplemented with independent measurements such as anglelap/stain, Hall effect, sheet resistance, saturation current (pulsed and dc [5]), and the dc properties of MESFET's. Only by insisting on self-consistency within some such highly redundant set of measurements can one confidently characterize the film.

\section{APPENDIX}

The specific combinations of Kelvin functions which appear in (16) and (17) can be represented by the following ascending series [18]:

$$
\begin{gathered}
\operatorname{ber}^{2}(x)+\operatorname{bei}^{2}(x)=\sum_{k=0}^{\infty} \frac{\left(\frac{1}{4} x^{2}\right)^{2 k}}{(k !)^{2}(2 k) !} \\
\text { ber }(x) \operatorname{bei}^{\prime}(x)-\operatorname{bei}(x) \operatorname{ber}^{\prime}(x)=\frac{x}{2} \sum_{k=0}^{\infty} \frac{\left(\frac{1}{4} x^{2}\right)^{2 k}}{(k !)^{2}(2 k+1) !} \\
\text { ber }(x) \operatorname{ber}^{\prime}(x)+\text { bei }(x) \operatorname{bei}^{\prime}(x)=\frac{1}{x} \sum_{k=1}^{\infty} \frac{\left(\frac{1}{4} x^{2}\right)^{2 k}}{(k !)^{2}(2 k-1) !} .
\end{gathered}
$$

Inserting these expansions into (15) gives

$$
\operatorname{Re}(Y) \approx \omega^{2} R_{e} C^{2}\left[\frac{1+\frac{1}{384} x_{a}^{4}+\frac{1}{1105920} x_{a}^{8}+\cdots}{1+\frac{1}{32} x_{a}^{4}+\frac{1}{24576} x_{a}^{8}+\cdots}\right]
$$

and

$$
\operatorname{Im}(Y) \approx j \omega C\left[\frac{1+\frac{1}{96} x_{a}^{4}+\frac{1}{122880} x_{a}^{8}+\cdots}{1+\frac{1}{32} x_{a}^{4}+\frac{1}{24576} x_{a}^{8}+\cdots}\right]
$$

where, as before, $x_{a}$ is defined by (12). Using (12) to eliminate $x_{a}$, and keeping only terms through $x_{a}^{4}$ gives

$$
\operatorname{Re}(Y) \approx \omega^{2} R_{e} C^{2}\left[\frac{1+\frac{1}{6} \omega^{2} R_{e}^{2} C^{2}+\cdots}{1+2 \omega^{2} R_{e}^{2} C^{2}+\cdots}\right]
$$

and

$$
\operatorname{Im}(Y) \approx j \omega C\left[\frac{1+\frac{2}{3} \omega^{2} R_{e}^{2} C^{2}+\cdots}{1+2 \omega^{2} R_{e}^{2} C^{2}+\cdots}\right] .
$$

In the same approximation, $\tan \phi$ is given by

$$
\tan \phi \approx \frac{1}{\omega R_{e} C}\left[\frac{1+\frac{2}{3} \omega^{2} R_{e}^{2} C^{2}+\cdots}{1+\frac{1}{6} \omega^{2} R_{e}^{2} C^{2}+\cdots}\right] .
$$

Equations (A6) and (A7) are only useful for $\omega R_{e} C<1$ and are about 5 percent high at $\omega R_{e} C=1$. Equation (A8) is 0.1 percent high at $\omega R_{e} C=1$. These equations give the secondorder corrections to the simple lumped-element equivalent circuit used in [11]. It is seen that as $\omega R_{e} C \rightarrow 0,(21)$ is obtained for $Z$, and the lumped model is recovered.

For large values of their arguments, the Kelvin functions are more conveniently represented by the following asymptotic expansions [18] :

$$
\operatorname{ber}^{2}(x)+\operatorname{bei}^{2}(x) \sim \frac{e^{x \sqrt{2}}}{2 \pi x}\left[1+\frac{1}{4 \sqrt{2}} \frac{1}{x}+\cdots\right]
$$

$$
\operatorname{ber}(x) \text { bei' }^{\prime}(x)-\text { bei }(x) \text { ber' }^{\prime}(x) \sim \frac{e^{x \sqrt{2}}}{2 \pi x}\left[\frac{1}{\sqrt{2}}+\frac{1}{8 x}+\cdots\right]
$$

and

$$
\operatorname{ber}(x) \text { ber' }^{\prime}(x)+\text { bei }(x) \text { bei }^{\prime}(x) \sim \frac{e^{x \sqrt{2}}}{2 x}\left[\frac{1}{\sqrt{2}}-\frac{3}{8 x}+\cdots\right]
$$

These expansions were used to obtain the limiting form of $Z$ as $\omega R_{e} C \rightarrow \infty$, given by (22). 


\section{ACKNOWLEGMENT}

The author wishes to thank Prof. H. Guckel for numerous helpful discussions, and for suggestions concerning the limiting behavior of distributed transmission lines.

\section{REFERENCES}

[1] J. Hilibrand and R. D. Gold, RCA Rev., vol. 21, p. 245, 1960.

[2] R. A. Moline, J. Appl. Phys., vol. 42, p. 3553, 1971.

[3] P. J. Baxandall, D. J. Colliver, and A. F. Fray, J. Phys. E, vol. 4, p. 213,1971.

[4] G. L. Miller, IEEE Trans. Electron Devices, vol. ED-19, p. 1103, 1971.

[5] P. L. Hower, W. W. Hooper, B. R. Cairns, R. D. Fairman, and D. A. Tremere, "The GaAs fieldeffect transistor" in Semicon" ductors and Semimetals, vol. 7a, R. K. Willardson and A. C. Beer, Eds. New York: Academic Press, 1971, ch. 3.

[6] D. P. Kennedy, P. C. Murley, and W. Kleinfelder, IBM J. Rei. Develop., vol. 12 , p. 399,1968

[7] D. P. Kennedy and R. R. O'Brian, IBM J. Res. Develop., vol. 1'3, p. $212,1969$.

[8] W. C. Johnson and P. T. Panousis, IEEE Trans. Electron Device?, vol. ED-18, p. $965,1971$.
[9] A. M. Goodman, J. Appl. Phys., vol. 34, p. 329, 1963.

[10] J. A. Copeland, IEEE Trans. Electron Devices, vol. ED-17, p. 404,1970

[11] J. D. Wiley and G. L. Miller, IEEE Trans. Electron Devices, vol. ED-22, p. 265, 1975.

[12] K. Lehovec, Appl. Phys. Lett., 26, p. 82, 1975.

[13] P. Kramer, C. de Vries, and L. J. van Ruyven, J. Electrochem Soc., vol. 122, p. 314, 1975 .

[14] R. N. Ghose, Microwave Circuit Theory and Analysis. New York: McGraw-Hill, 1963, ch. 12

[15] E. H. Nicollian and A. Goetzberger, IEEE Trans. Electron Devices, vol. ED-12, p. 108, 1965.

[16] K. Lehovec, Appl. Phys. Lett., vol. 25, p. 279, 1974.

[17] K. Lehovec, A. Fedotowsky, and D. W. Crain, Solid State Electron., vol. 19 , p. $249,1976$.

[18] Handbook of Mathematical Functions, M. Abramowitz and I. A. Stegun, Eds. Washington, DC: Government Printing Office, 1964, ch. 9.

[19] K. Lehovec and R. Zuleeg, presented at the Fifth Intl. Symp. GaAs and Related Compounds (Deauville, France, Sept. 24-26, 1974). Proceedings published by the Institute of Physics as Inst. Phys. Conf., ser. 24, ch. 5, 1975.

[20] J. D. Wiley, unpublished work.

[21] E. Wasserstrom and J. McKenna, Bell Syst. Tech. J., pp. 853877, May, June, 1970. 